\title{
Acute Pancreatitis Complicating Intragastric Balloon Insertion
}

\author{
AbdulRahman Elsheikh Mohammed Ali Benmousa \\ Division of Gastroenterology, Department of Medicine, Riyadh Military Hospital, \\ Riyadh, Saudi Arabia
}

\section{Key Words}

Intragastric balloon · Obesity · Acute pancreatitis

\begin{abstract}
We present a case of acute pancreatitis that happened as a complication of intragastric balloon insertion used for weight reduction in a young obese lady. Although many complications can happen with this type of procedure, this presentation is very rare and may not have been reported previously. The patient responded well to removal of the balloon since her pain resolved completely and her serum amylase normalized. She did not have any obvious cause of pancreatitis other than a significant radiologically proven compression of the pancreas by the balloon.
\end{abstract}

\section{Introduction}

Intragastric balloons are becoming an increasingly used modality for weight reduction in obese patients. Although endoscopic placement of an intragastric balloon (Garron-Edwards Gastric Bubble) was discontinued in 1987 because of a high complication rate, and similar finding in our department of frequent and serious complication and ineffectiveness regarding weight loss had led to stopping the use of balloon for weight reduction [1], more recent trials have shown better tolerability and a better safety profile of the procedure with the use of saline-filled Bioenteric Intragastric Balloon (BIB) System [2].

Many side effects have been reported after BIB insertion and they are mostly related to the pressure effect and rupture of the balloon. We present an interesting and rare complication of intragastric balloon used for treatment of obesity in one of our patients. Our department restarted insertion of BIB in June 2005 and till now over 135 balloons have been inserted. The tolerance rate is high $(>90 \%)$ and that is mostly related to good information of the patients about the side effects of the procedure and to proper management of their symptoms after the procedure. Only one of these 135 patients had serious complications as reported below.

Dr. AbdulRahman Elsheikh Mohammed
Division of Gastroenterology, Department of Medicine, Riyadh Military Hospital PO Box 7897, Riyadh 11159 (Saudi Arabia)

Fax+966 1475 7859, E-Mail alibenmousa@hotmail.com 


\section{Case Presentation}

Our patient is a 31-year-old female nurse who was seen in the gastroenterology clinic for management of obesity after failing to reduce weight by diet and exercise. Her weight at presentation was $85 \mathrm{~kg}$ and her height was $162 \mathrm{~cm}$ with a BMI of 33.2. She was complaining of backache and knee pain which were attributed to osteoarthritic changes secondary to obesity. The patient had BIB inserted in the gastric fundus and filled with $600 \mathrm{ml}$ of saline on 20th of November 2006. She tolerated the procedure initially and lost $16 \mathrm{~kg}$ over 75 days. She presented to the clinic ten weeks after BIB placement complaining of sudden acute severe epigastric pain and repeated vomiting. Examination revealed fullness in the epigastric area and tenderness; bowel sounds were present.

Her initial laboratory tests showed normal full blood count, electrolytes and hepatic and renal profile. Her serum calcium was $2.2 \mathrm{mmol} / \mathrm{l}$ (normal 2.0-2.5), cholesterol was $5.5 \mathrm{mmol} / \mathrm{l}$ (normal <5.2) and triglyceride was $2.2 \mathrm{mmol} / \mathrm{l}$ (normal 0.45-1.7). Serum amylase was $502 \mathrm{U} / \mathrm{l}$, which was more than three times the upper limit of normal (normal 0-100).

The patient had emergency ultrasound of the abdomen which showed that the intragastric balloon was indenting the tail of the pancreas, but no peripancreatic fluid was noted. There were no gallstones and no evidence of biliary obstruction as the common bile duct diameter was $3 \mathrm{~mm}$ and no intrahepatic biliary dilatation was seen. A CT scan of the abdomen (fig. 1) was done and revealed a similar finding of a compressed body of the pancreas by distended stomach; the compression was exaggerated in the supine position. The patient has no history of alcohol consumption or use of medications known to precipitate pancreatitis.

In view of the severity of symptoms, high amylase level and the ultrasound and CT scan findings, she was diagnosed as having acute pancreatitis attributed to the balloon. According to Ranson's criteria her score at presentation was 0 , and since there was no necrosis on CT scan it was graded as mild acute pancreatitis.

Since the patient was symptomatic, she had an upper gastrointestinal endoscopy which showed a normal appearing esophagus, stomach and duodenum. During this procedure the intragastric balloon was removed from the gastric fundus on 6th of February 2007. The patient's symptoms improved significantly and her amylase level came back to normal. The final diagnosis was mild acute pancreatitis caused by BIB insertion.

\section{Discussion}

$\mathrm{BIB}$ is a nonsurgical procedure that has been used in treating obesity and morbid obesity. It works by restriction of gastric intake. It is less invasive and cheaper than bariatric surgery but can only be left in the stomach for six months. The efficacy of this procedure is variable according to various clinical trials. While in one trial a 11.2 to $12.8 \mathrm{~kg}$ loss of weight after 3 months and a 16.7 to $20.0 \mathrm{~kg}$ loss after 6 months of balloon treatment was achieved [2], others showed only $5.9 \mathrm{~kg}(7.5 \%)$ loss of starting body weight and $31 \%$ of excess weight [3] 6 months after balloon placement.

Although endoscopic placement of BIB is considered safe and relatively easy compared to surgical treatment of obesity, patient tolerance remains one of the most important limitations for the use of this treatment modality. The majority of adverse effects of intragastric balloon are related to gastric distension and reduced gastric capacity, and the incidence of symptoms (nausea, vomiting, abdominal cramps) is directly related to balloon size $[4,5]$. More serious adverse events have been reported including ulcers or erosions caused by the pressure on the gastric mucosa, tear as a result of improper placement of the balloon and migration of a leaking balloon causing obstruction of the gut. The incidence of mucosal injury is $4 \%$ and premature deflation of the balloon is required in 7-8\%, although much higher rates were reported from an Asian group [3, 6]. Stomach perforation and severe esophagitis have also been reported [7-10]. 
In spite of the close relation of the stomach to the pancreas, we are not aware of any reported case of acute pancreatitis in relation to BIB insertion. Probably it is because of the site of the balloon which is usually located at the fundus of the stomach rather than the antrum. One case was reported of incomplete bowel obstruction and pancreatitis following dislodgement of the intragastric balloon [11].

Although our patient had her balloon placed in a good position, she still developed acute pancreatitis evident both clinically (epigastric pain and vomiting after a period of good tolerability of the balloon) and biochemically (hyperamylasemia) with radiological evidence of pressure effect on the pancreas. The improvement of her symptoms and return of amylase level to normal after BIB removal further adds to this diagnosis. Pancreatitis developed in this patient more than 2 months after insertion of the BIB; whether there was continuous pressure on the pancreas from the beginning or whether the balloon settled more in the fundus and caused pressure and irritation on the pancreas is difficult to determine at this stage. It is important to mention that the most common causes of acute pancreatitis, namely gallstones and alcohol, were excluded in this patient. Our previous observations have shown a low incidence of alcoholic pancreatitis in Saudi Arabia [12] that is probably related to alcohol prohibition in this country. $66 \%$ of 120 acute pancreatitis cases presented to our hospital were biliary in origin and $6 \%$ of them were due to alcohol [13]. Many factors contribute to the development of acute alcohol-induced pancreatitis [14] and attacks can be recurrent with significant risk of chronic pancreatitis.

The severity and prognosis of acute pancreatitis depend on particular prognostic systems or markers which include the calculation of positive and negative likelihood ratios derived from combination of sensitivity and specificity, and applying these parameters will be affected by pretest probability of the disease [15]. Luckily this patient had a mild attack of acute pancreatitis which responded well to removal of the causative agent and observation. In a severer form of acute pancreatitis many complications can occur, including pancreatic necrosis, infection, pseudocyst and retroperitoneal collection $[16,17]$.

We believe this is the first case report of acute pancreatitis following BIB insertion without displacement of the balloon. Reporting this case might increase physicians' awareness of this complication. Since symptoms of pancreatitis can be misinterpreted as the effect of gastric distension by the balloon, this diagnosis can be missed in some patients, leading to more serious sequale and complications of pancreatitis. 
Case Reports in | Case Rep Gastroenterol 2008;2:291-295 Gastruenterology

Fig. 1. Abdominal CT scan showing intragastric compression of the tail of the pancreas.

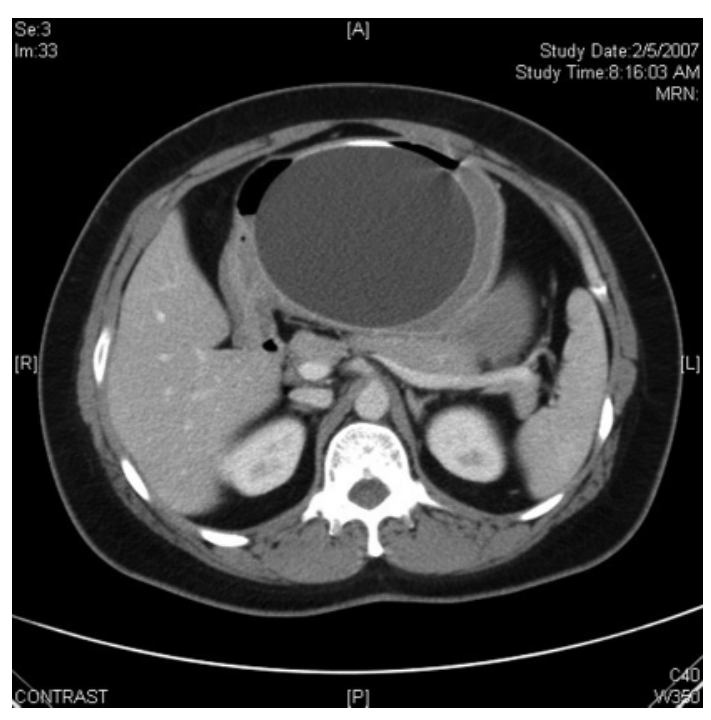




\section{References}

1 Alkarawi MA, Aljumaah AA, Sheail G, Mohammed AE: Intragastric balloon in the treatment of obesity. Saudi Med J 1992;13:393-394.

2 Mathus-Vliegen EM, Tytgat GNJ: Intragastric balloon for treatment-resistant obesity: Safety, tolerance, and efficacy of 1-year balloon treatment followed by a 1-year balloon-free follow-up. Gastrointest Endosc 2005;61:19-27.

-3 Ganesh R, Rao AD, Baladas HG, Leese T: The Bioenteric Intragastric Balloon (BIB) as a treatment for obesity: poor results in Asian patients. Singapore Med J 2007;48:227-231.

4 Geliebter A, Westreich S, Gage D: Gastric distention by balloon and test-meal intake in obese and lean subjects. Am J Clin Nutr 1988;48:592-594.

5 Geliebter A: Gastric distension and gastric capacity in relation to food intake in humans. Physiol Behav 1988;44:665-668.

6 Mathus-Vliegen EM: Treating morbid and supermorbid obesity. Int J Gastroenterol 2000;5:9-12.

$\checkmark 7$ Roche-Nagle G, Mulligan E, Connolly E, Lane B: Unusual cause of a perforated stomach. Ann R Coll Surg Engl 2003;85:396-397.

$>8$ Laurent B, Charles D, Laurent M: Covered gastric perforation by the BioEnterics Intragastric Balloon. J Clin Gastroenterol 2001;33:344-345.

-9 Genco A, Bruni T, et al: Bioenteric Intragastric Balloon: the Italian experience with 2,515 patients. Obes Surg 2005;15:1161-1164.

10 Sallet JF: Intragastric Balloon. Endoscopic gastroplasty for the treatment of obesity. Americana, Caminho Editorial, 2002.

11 Holland S, Bach D, Duff J: Balloon therapy for obesity - when the balloon bursts. J Can Assoc Radiol 1985;36:347-349.

12 Hamid MA, AlKarawi MA, Mohamed AE: Acute pancreatitis in alcohol prohibited society. JIMA 1989;21:60-63.

13 Pandol SJ, Raraty M: Pathobiology of alcoholic pancreatitis. Pancreatology 2007;7:105-114.

14 Al Shehri MA, Mohamed AE, Bushnak MA, AlKarawi MA: Acute pancreatitis; six and a half year experience. Saudi Med J 1992;131:46-48.

15 Windsor JA: Assessment of the severity of acute pancreatitis: no room for complacency. Pancreatology 2008;8:105-109.

16 Lawson TL: Acute pancreatitis and its complications. Computed tomography and sonography. Radiol Clin North Am 1983;21:495-513.

17 Tai SS, Foo NP, Lin HJ, Tseng JC: Severe complication of pancreatitis - huge retroperitoneal abscess formation. Pancreatology 2007;7:86-87. 